\title{
Algorithm based on Histogram and Entropy for Edge Detection in Gray Level Images
}

\author{
Mohamed A. El-Sayed \\ Department of Mathematics, Faculty of Science, University of Fayoum, Egypt. \\ Assistant professor, Dept of CS, College of CIT, Taif University, KSA \\ mas06@fayoum.edu.eg
}

\begin{abstract}
Edge detection and feature extraction are widely used in image processing and computer vision applications. Most of the traditional methods for edge detection are based on the first and second order derivatives of gray levels of the pixels of the original image utilizing 2D spatial convolution masks to approximate the derivative. In this paper we present an algorithm for edge detection in gray level images. The main objective is to solve the previous problem of traditional methods with generate suitable quality of edge detection. Our new algorithm is based on two definitions of entropy: Shannon's classical concept and a variation called Tsallis entropy. The novel approach utilizing Subextensive Tsallis entropy rather than the evaluation of derivatives of the image in detecting edges in gray level images has been proposed. Here, we have used a suitable threshold value to segment the image and achieve the binary image. The effectiveness is demonstrated by using many different kinds of test images from the real-world and synthetic images. The results of this study were quite promising.
\end{abstract}

\section{terms/Keywords}

Algorithms, Automatic thresholding, Edge detection, Tsallis entropy

\section{SUBJECT CLASSIFICATION}

AMS Classification: 68U10, 62H35, 94A17.

\section{TYPE (METHOD/APPROACH)}

Quasi-Experimental; Literary Analysis; Survey/Interview

\section{Council for Innovative Research}

Peer Review Research Publishing System

Journal: International Journal of Computers \& Technology

Vol 11, No.1

editor@cirworld.com

www.cirworld.com, member.cirworld.com 


\section{INTRODUCTION}

Edge detection is a crucial step in edge extraction and object delineation in image processing. An effective edge detector reduces a large amount of data but still keeps most of the important feature of the image. As one of the first-use methods, edge detection is widely used in imagery data processing such as remote sensing data processing. For example, for an aerial image, a high quality edge detector not only obtains a good understanding of the image but also provides a simple but significant input for the process of extracting edges of some specific objects. So, edge detection has received much attention during the past two decade because of its significant importance in many research areas [2,14]. The detection results benefit applications such as image enhancement, recognition, morphing, compression, retrieval, watermarking, hiding, restoration and registration etc. Edge detection concerns localization of abrupt changes in the gray level of an image. Edge detection can be defined as the boundary between two regions separated by two relatively distinct gray level properties. The causes of the region dissimilarity may be due to some factors such as the geometry of the scene, the radio metric characteristics of the surface, the illumination and so on [10].

Most of the classical methods for edge detection based on the derivative of the pixels of the original image are Gradient operators, Laplacian and Laplacian of Gaussian operators [13]. Gradient based edge detection methods, such as Roberts, Sobel and Prewitts, have used two 2-D linear filters to process vertical edges and horizontal edges separately to approximate first-order derivative of pixel values of the image. The Laplacian edge detection method has used a 2-D linear filter to approximate second-order derivative of pixel values of the image [15]. Major drawback of second-order derivative approach is that the response at and around the isolated pixel is much stronger. The Laplacian generally is not used in its original form for edge detection for several reasons: As a second-order derivative, the Laplacian typically is unacceptably sensitive to noise. The magnitude of the Laplacian produces double edges, an undesirable effect because it complicates segmentation. For these reasons, the Laplacian is combined with smoothing as a precursor to finding edges via zerocrossings. Marr and Hildreth achieved this by using the Laplacian of a Gaussian (LOG) function as a filter [6]. The paper [7] classified and comparative study of edge detection algorithms are presented. Experimental results prove that Boie-Cox, Shen- Castan and Canny operators are better than Laplacian of Gaussian (LOG), while LOG is better than Prewitt and Sobel in case of noisy image.

To solve these problems, the study proposed a novel approach based on information theory. Tsallis entropy is the most important among several measures of information. Edges can be extracted by the detection of all pixels on the borders between different homogenous areas. Entropy measures the randomness of intensity distribution [3]. According to this property of entropy, the value of entropy is low for homogenous areas and is high where the diversity of gray level of pixels is large [11]. The proposed method is decrease the computation time.

This paper is organized as follows: in Section 2 presents some fundamental concepts of the mathematical setting of the threshold selection and Tsallis entropy. Section 3, we describe the newly proposed method of edge detection in gray level images using a Tsallis entropy. In Section 4, we report the effectiveness of our method when applied to some real-world and synthetic images. In Section 5, we present some concluding remarks about our method.

\section{SELECTION OF THRESHOLD VALUE}

Entropy is a concept in information theory. Entropy is used to measure the amount of information [8]. Entropy is defined in terms of the probabilistic behavior of a source of information. In accordance with this definition, a random event $E$ that occurs with probability $P(E)$ is said to contain $I(E)=\log (1 / P(E))=-\log (P(E))$ units of information. The amount $I(E)$ is called the self-information of event $E$ or information content of $E$. The amount of self information of the event is inversely related to its probability. If $P(E)=1$, then $I(E)=0$ and no information is attributed to it. In this case, uncertainty associated with the event is zero. Thus, if the event always occurs, then no information would be transferred by communicating that the event has occurred. If $P(E)=0.8$, then some information would be transferred by communicating that the event has occurred [10]. The base of the logarithm determines the unit which is used to measure the information. If the base of the logarithm is 2 , then unit of information is bit. If $P(E)=1 / 2$, then $I(E)=-\log _{2}(1 / 2)=1$ bit. That is, 1 bit is the amount of information conveyed when one of two possible equally likely events occurs. A simple example of such a situation is flipping a coin and communicating the result (Head or Tail).

The set $Z$ of source symbols is referred to as the source alphabet, $Z=\left\{s_{1}, s_{2}, s_{3}, \ldots, s_{k}\right\}$. The set of all source symbol probabilities is denoted by $P, P=\left\{p_{1}, p_{2}, p_{3}, \ldots, p_{k}\right\}$. This set of probabilities must satisfy the condition $\sum_{i=1}^{k} p_{i}=1$,

$0 \leq p_{i} \leq 1$. The average information per source output, denoted $S(Z)$ [5], is: $S(Z)=-\sum_{i=1}^{k} p_{i} \ln \left(p_{i}\right)$, being $k$ the total number of states.

If we consider that a system can be decomposed in two statistical independent subsystems $A$ and $B$, the Shannon entropy has the extensive property $S(A+B)=S(A)+S(B)$. This formalism has been shown to be restricted to the BoltzmannGibbs-Shannon (BGS) statistics.

However, for non-extensive systems, some kind of extension appears to become necessary. Tsallis [12] has proposed a generalization of the BGS statistics which is useful for describing the thermo statistical properties of non-extensive systems. It is based on a generalized entropic form, 


$$
S_{q}=\frac{1}{q-1}\left(1-\sum_{i=1}^{k} p_{i}^{q}\right)
$$

where the real number $q$ is a entropic index that characterizes the degree of non-extensivity. This expression recovers to BGS entropy in the limit $q \rightarrow 1$.Tsallis entropy has a non-extensive property for statistical independent systems, defined by the following rule [1] :

$$
S_{q}(A+B)=S_{q}(A)+S_{q}(B)+(1-q) \cdot S_{q}(A) \cdot S_{q}(B) .
$$

Let $f(x, y)$ be the gray value of the pixel located at the point $(x, y)$. In a digital image $\{f(x, y) \mid x \in\{1,2, \ldots, M\}, y \in\{1,2, \ldots, N\}\}$ of size $M \times N$, let the histogram be $h(a)$ for $a \in\{0,1,2, \ldots, 255\}$ with $f$ as the amplitude (brightness) of the image at the real coordinate position $(x, y)$. For the sake of convenience, we denote the set of all gray levels $\{0,1,2, \ldots, 255\}$ as $G$. Global threshold selection methods usually use the gray level histogram of the image. The optimal threshold $t^{*}$ is determined by optimizing a suitable criterion function obtained from the gray level distribution of the image and some other features of the image.

Let $t$ be a threshold value and $B=\left\{b_{0}, b_{1}\right\}$. be a pair of binary gray levels with $\left\{b_{0}, b_{1}\right\} \in G$. Typically $b_{0}$ and $b_{1}$ are taken to be 0 and 1 , respectively. The result of thresholding an image function $f(x, y)$ at gray level $t$ is a binary function $f_{t}(x, y)$ such that $f_{t}(x, y)=b_{0}$ if $f_{t}(x, y) \leq t$ otherwise, $f_{t}(x, y)=b_{1}$. In general, a thresholding method determines the value $t^{*}$ of $t$ based on a certain criterion function. If $t^{*}$ is determined solely from the gray level of each pixel, the thresholding method is point dependent [9].

Let $p_{i}=p_{1}, p_{2}, \ldots, p_{k}$ be the probability distribution for an image with $k$ gray-levels. From this distribution, we derive two probability distributions, one for the object (class $A$ ) and the other for the background (class $B$ ), given by:

$$
p_{A}: \frac{p_{1}}{P_{A}}, \frac{p_{2}}{P_{A}}, \ldots, \frac{p_{t}}{P_{A}} \text { and } p_{B}: \frac{p_{t+1}}{P_{B}}, \frac{p_{t+2}}{P_{B}}, \ldots, \frac{p_{k}}{P_{B}} \text { where } P_{A}=\sum_{i=1}^{t} p_{i}, P_{B}=\sum_{i=t+1}^{k} p_{i}
$$

The Tsallis entropy of order $q$ for each distribution is defined as:

$$
S_{q}^{A}(t)=\frac{1}{q-1}\left(1-\sum_{i=1}^{t} p_{A}^{q}\right) \text { and } S_{q}^{B}(t)=\frac{1}{q-1}\left(1-\sum_{i=t+1}^{k} p_{B}^{q}\right)
$$

The Tsallis entropy $S_{q}(t)$ is parametrically dependent upon the threshold value $t$ for the foreground and background. It is formulated as the sum each entropy, allowing the pseudo -additive property, defined in equation (2). We try to maximize the information measure between the two classes (object and background). When $S_{q}(t)$ is maximized, the luminance level $t$ that maximizes the function is considered to be the optimum threshold value [4].

$$
t^{*}(q)=\underset{t \in G}{\operatorname{Arg} \max }\left[S_{q}^{A}(t)+S_{q}^{B}(t)+(1-q) \cdot S_{q}^{A}(t) \cdot S_{q}^{B}(t)\right]
$$

Considering $S_{q} \geq 0$ in the pseudo-additive formalism of Eq. (4), three different entropies can be defined with regard to different values of $q$. For $q<1$, the Tsallis entropy becomes a "subextensive entropy" where $S_{q}(A+B)<S_{q}(A)+S_{q}(B)$; for $q=1$, the Tsallis entropy reduces to an standard "extensive entropy" where $S_{q}(A+B)=S_{q}(A)+S_{q}(B)$; for $q>1$, the Tsallis entropy becomes a "superextensive entropy" where $S_{q}(A+B)>S_{q}(A)+S_{q}(B)$.

In the proposed scheme, first create a binary image by choosing a suitable threshold value using Tsallis entropy. The technique consists of treating each pixel of the original image and creating a new image, such that $f_{t}(x, y)=0$ if $f_{t}(x, y) \leq t^{*}(q)$ otherwise, $f_{t}(x, y)=1$ for every $x \in\{1,2, \ldots, M\}, y \in\{1,2, \ldots, N\}$.

Theorem 1: The threshold value equals to the same value found by Shannon's method when $q \rightarrow 1$.

Proof: The limiting case of the proposed extension is Shannon's method. To see this, compute the limiting value of $S_{q}^{A}(t)$ and $S_{q}^{B}(t)$ as $q \rightarrow 1$. Hence, 


$$
\begin{aligned}
\lim _{q \rightarrow 1} S_{q}^{A+B}(t) & =\lim _{q \rightarrow 1}\left[S_{q}^{A}(t)+S_{q}^{B}(t)+(1-q) \cdot S_{q}^{A}(t) \cdot S_{q}^{B}(t)\right] . \\
= & \lim _{q \rightarrow 1}\left[S_{q}^{A}(t)+S_{q}^{B}(t)\right] . \\
= & \lim _{q \rightarrow 1}\left[\frac{1}{q-1}\left(1-\sum_{i=0}^{t}\left(p_{A}\right)^{q}\right)\right]+\lim _{q \rightarrow 1}\left[\frac{1}{q-1}\left(1-\sum_{i=t+1}^{255}\left(p_{B}\right)^{q}\right)\right] \\
& =-\lim _{q \rightarrow 1} \frac{d}{d q}\left(\sum_{i=0}^{t}\left(p_{A}\right)^{q}\right)-\lim _{q \rightarrow 1} \frac{d}{d q}\left(\sum_{i=t+1}^{255}\left(p_{B}\right)^{q}\right) \\
& =-\lim _{q \rightarrow 1} \sum_{i=0}^{t} \frac{d}{d q}\left(p_{A}\right)^{q}-\lim _{q \rightarrow 1} \sum_{i=t+1}^{255} \frac{d}{d q}\left(p_{B}\right)^{q}
\end{aligned}
$$

but, $\frac{d}{d q}\left(a^{q}\right)=e^{q \ln a} \cdot \ln (a)$, i.e. $\lim _{q \rightarrow 1} \frac{d}{d q}\left(a^{q}\right)=e^{\ln (a)} \cdot \ln (a)=\operatorname{a} \ln (a)$

hence $\lim _{q \rightarrow 1} S_{q}^{A+B}(t)=-\sum_{i=0}^{t} p_{A} \ln \left(p_{A}\right)-\sum_{i=t 1}^{255} p_{B} \ln \left(p_{B}\right)$

$$
=S^{A}(t)+S^{B}(t)
$$

Hence, when $q \rightarrow 1$, the threshold value in Equation (1), equals to the same value found by Shannon's method. Thus this proposed method includes Shannon's method as a special case. The following expression can be used as a criterion function to obtain the optimal threshold at $q \rightarrow 1$.

$$
t^{*}(1)=\underset{t \in G}{\operatorname{Arg} \max }\left[S^{A}(t)+S^{B}(t)\right]
$$

The Tsallis Threshold procedure to select suitable threshold value $t^{*}$ and $q$ can now be described as follows: Procedure TsallisThreshold,

Input: A digital grayscale image $A$ of size $M \times N$.

Output: The suitable threshold value $t^{*}$ of $A$, for $q \geq 0$.

Begin

1. Let $f(x, y)$ be the original gray value of the pixel at the point $(x, y), x=1 . . M, y=1 . . N$.

2. Calculate $\mathrm{pi}$ and $\mathrm{P}$ be the probability distribution, $i=0,1, \ldots, 255$.

3. For all $t \in\{0,1, \ldots, 255\}$,

i. Calculate $P_{A}=\sum_{i=1}^{t} p_{i}$, and $P_{B}=\sum_{i=t+1}^{255} p_{i}$, then calculate $p_{A}$ and $p_{B}$.

ii. If $q \neq 1$ Then $t^{*}(q)=\underset{t \in G}{\operatorname{Arg} \max }\left[S_{q}^{A}(t)+S_{q}^{B}(t)+(1-q) \cdot S_{q}^{A}(t) \cdot S_{q}^{B}(t)\right]$.

Else $t^{*}(1)=\underset{t \in G}{\operatorname{Arg} \max }\left[-\sum_{i=1}^{t} p_{i} \ln \left(p_{i}\right)-\sum_{i=t+1}^{255} p_{i} \ln \left(p_{i}\right)\right]$.

Endlf.

Endfor End.

\section{The Edge Detection:}

We will use the usual masks for detecting the edges. A spatial filter mask may be defined as a matrix $w$ of size $m \times n$. Assume that $m=2 \alpha+1$ and $n=2 \beta+1$, where $\alpha, \beta$ are nonzero positive integers. For this purpose, smallest meaningful size of 
the mask is $3 \times 3$. Such mask coefficients, showing coordinate arrangement as Figure 1.a . Image region under the above mask is shown as Figure 1.b.

\begin{tabular}{|c|c|c|c|c|c|c|c|c|}
\hline$w(-1,-1)$ & $w(-1,0)$ & $w(-1,1)$ & $f(x-1, y-1)$ & $f(x-1, y)$ & $f(x-1, y+1)$ & 1 & 1 & 1 \\
\hline$w(0,-1)$ & $w(0,0)$ & $w(0,1)$ & $f(x, y-1)$ & $f(x, y)$ & $f(x, y+1)$ & 1 & $x$ & 1 \\
\hline$w(1,-1)$ & $w(1,0)$ & $w(1,1)$ & $f(x+1, y-1)$ & $f(x+1, y)$ & $f(x+1, y+1)$ & 1 & 1 & 1 \\
\hline
\end{tabular}

Figure 1.a

Figure 1.b

Figure 1.c

In order to edge detection, firstly classification of all pixels that satisfy the criterion of homogeneousness, and detection of all pixels on the borders between different homogeneous areas. In the proposed scheme, first create a binary image by choosing a suitable threshold value using Tsallis entropy. Window is applied on the binary image. Set all window coefficients equal to 1 except centre, centre equal to $x$ as shown in Figure 1.c.

Move the window on the whole binary image and find the probability of each central pixel of image under the window. Then, the entropy of each central pixel of image under the window is calculated as:

$$
S(C P i x)=-p_{c} \ln \left(p_{c}\right)
$$

Where, pc is the probability of central pixel CPix of binary image under the window. When the probability of central pixel, $p_{c}=1$, then the entropy of this pixel is zero. Thus, if the gray level of all pixels under the window homogeneous, $p_{c}=1$ and $S=0$. In this case, the central pixel is not an edge pixel. Other possibilities of entropy of central pixel under window are shown in Table 1.

Table 1: $p$ and $S$ of central under window

\begin{tabular}{|c|c|c|c|c|c|c|c|c|c|}
\hline$p$ & $1 / 9$ & $2 / 9$ & $3 / 9$ & $4 / 9$ & $5 / 9$ & $6 / 9$ & $7 / 9$ & $8 / 9$ & $9 / 9$ \\
\hline \hline$S$ & 0.2441 & 0.3342 & 0.3662 & 0.3604 & 0.3265 & 0.2703 & 0.1955 & 0.1047 & 0.0 \\
\hline
\end{tabular}

In cases $p_{c}=8 / 9$, and $p_{c}=7 / 9$, the diversity for gray level of pixels under the window is low. So, in these cases, central pixel is not an edge pixel. In remaining cases, $\mathrm{p} c \leq 6 / 9$, the diversity for gray level of pixels under the window is high. The complete TsallisEdgeDetection algorithm can now be described as follows:

Algorithm TsallisEdgeDetection;

Input: A digital grayscale image $A$ of size $M \times N$.

Output: The edge detection image $g$ of $A$.

Begin

Step 1: Applying TsallisThreshold procedure to select suitable threshold value $t^{*}$ and $q$.

Step 2: Create a binary image:

For all $x, y$, If $f(x, y) \leq t^{*}$ then $f(x, y)=0$ Else $f(x, y)=1$.

Step 3: Create a mask, $w$, with dimensions $m \times n$ : Normally, $m=3$ and $n=3$.

$a=(m-1) / 2$ and $b=(n-1) / 2$.

Step 4: Create an $M \times N$ output image, $g$ : For all $x$ and $y$, Set $g(x, y)=f(x, y)$.

Step 5: Checking for edge pixels:

$$
\begin{aligned}
& \text { For } y=b+1 \text { to } N-b \\
& \text { For } x=a+1 \text { to } M-a \\
& \text { sum }=0 ; \\
& \text { For } k=-b \text { to } b \\
& \text { For } j=-a \text { to } a
\end{aligned}
$$


If $(f(x, y)=f(x+j, y+k))$ Then sum $=$ sum +1 End if

End for

End for

If ( sum >6) Then $g(x, y)=0$ Else $g(x, y)=1$ End if

End for

End for

End algorithm.

\section{ANALYSIS OF TEST RESULTS}

In this section, we discuss the experimental results obtained using the proposed method. The performance of the proposed scheme is evaluated through the simulation results using MATLAB for a set of sixteen test images and the results of the proposed scheme are compared with the results of well-established edge detection operator on the same set of test images. The resent published method by B. Singh and A. P. Singh [10] is chosen for comparison because both approaches are more similar in the technique of implementation. For this purpose, first group, a standard test images: bacteria.tif, blood1.tif, brain.tif, cameraman.tif, eight.tif, ic.tif, rice.tif; saturn.tif, and tree.tif were taken from MATLAB environment. second group, a test images from the real-world and synthetic images from other papers: analskin.tif, cell.tif, eggspindle.tif, pout.tif, rose.tif, synthetic.tif, and things.tif.

Here, we have used in addition to the original gray level function $f(x, y)$, a function $g(x, y)$ that is the average gray level value in a $3 \times 3$ neighborhood around the pixel $(x, y)$.

Table 2 The optimal threshold values $t^{*}$ for various values of $q$

\begin{tabular}{|c|c|c|c|c|c|c|c|c|c|c|c|c|}
\hline \multirow{2}{*}{ Test Image } & \multicolumn{12}{|c|}{$t^{*}(\ldots)$} \\
\hline & 0.05 & 0.1 & 0.2 & 0.3 & 0.4 & 0.5 & 0.9 & 1 & 1.1 & 1.65 & 1.7 & 3 \\
\hline analskin.tif & 121 & 132 & 74 & 74 & 74 & 58 & 58 & 58 & 247 & 247 & 30 & 0 \\
\hline bacteria.tif & 107 & 105 & 104 & 104 & 104 & 104 & 104 & 104 & 239 & 14 & 44 & 5 \\
\hline blood1.tif & 143 & 143 & 138 & 171 & 171 & 171 & 171 & 171 & 255 & 48 & 106 & 46 \\
\hline brain.tif & 121 & 132 & 133 & 140 & 141 & 155 & 164 & 172 & 255 & 24 & 148 & 0 \\
\hline cameraman.tif & 128 & 125 & 123 & 122 & 192 & 192 & 192 & 192 & 253 & 38 & 14 & 7 \\
\hline cell.tif & 124 & 124 & 121 & 121 & 117 & 117 & 113 & 113 & 247 & 46 & 3 & 2 \\
\hline coins.png & 161 & 159 & 158 & 157 & 158 & 159 & 200 & 210 & 255 & 140 & 82 & 56 \\
\hline eggspindle.tif & 91 & 97 & 115 & 115 & 115 & 121 & 121 & 121 & 205 & 85 & 25 & 0 \\
\hline ic.tif & 125 & 125 & 92 & 88 & 84 & 84 & 83 & 83 & 255 & 19 & 1 & 0 \\
\hline pout.tif & 152 & 158 & 161 & 162 & 166 & 166 & 166 & 167 & 224 & 89 & 99 & 73 \\
\hline rice.tif & 132 & 132 & 129 & 129 & 129 & 126 & 121 & 118 & 255 & 43 & 42 & 40 \\
\hline rose.tif & 129 & 132 & 138 & 142 & 156 & 158 & 158 & 103 & 255 & 28 & 17 & 0 \\
\hline saturn.tif & 131 & 134 & 141 & 146 & 158 & 160 & 165 & 165 & 255 & 36 & 1 & 0 \\
\hline synthetic.tif & 121 & 121 & 121 & 121 & 120 & 113 & 113 & 113 & 255 & 180 & 0 & 0 \\
\hline things.tif & 120 & 112 & 173 & 180 & 180 & 180 & 180 & 180 & 255 & 59 & 132 & 16 \\
\hline trees.tif & 130 & 130 & 126 & 125 & 125 & 124 & 124 & 124 & 255 & 23 & 40 & 0 \\
\hline
\end{tabular}

Our analysis is based on how much information is lost due to edge detection. In this analysis, given two thresholded images of a same original image, we prefer the one which lost the least amount of information. The optimal threshold value was computed by the proposed method for these sixteen images. Table 1 lists the optimal threshold values $t^{*}$ that are found for these images for q values equal to $0.05,0.1,0.2,0.3,0.4,0.5,0.9,1,1.1,1.65,1.7$ and 3 , respectively. The original images together with their edge images obtained by using the optimal threshold of some values $\mathrm{t}^{\star}$ are displayed side by side in Figs. 3-18.

Using the above sixteen images and also some other images, we conclude that when $q$ value lies between 0 and 1 , our proposed method produced good edge detection with optimal threshold values (see the first columns of Table 2 and Figure2). Moreover, the optimal threshold value does not change very much when the fractional $q$ value changes a little. 
However, in most cases, when q was greater than 1, this proposed method did not produce good edge images and the threshold values produced were unacceptable (see the last columns of Table 2 and Figure2). When the value of $q$ was one - Shannon entropy - the threshold value produced was not always a good edge images (see Figs. 3-18).

The results of edge detections for these test images using the method in [10] - Shannon entropy - and proposed scheme are shown in Figs. 3-18. From the results; it has again been observed that the performance of the proposed edge detection scheme is found to be satisfactory for all the test images as compare to the performance of the previous method. It has been observed that the proposed method for edge detection works well as compare to the previous method.

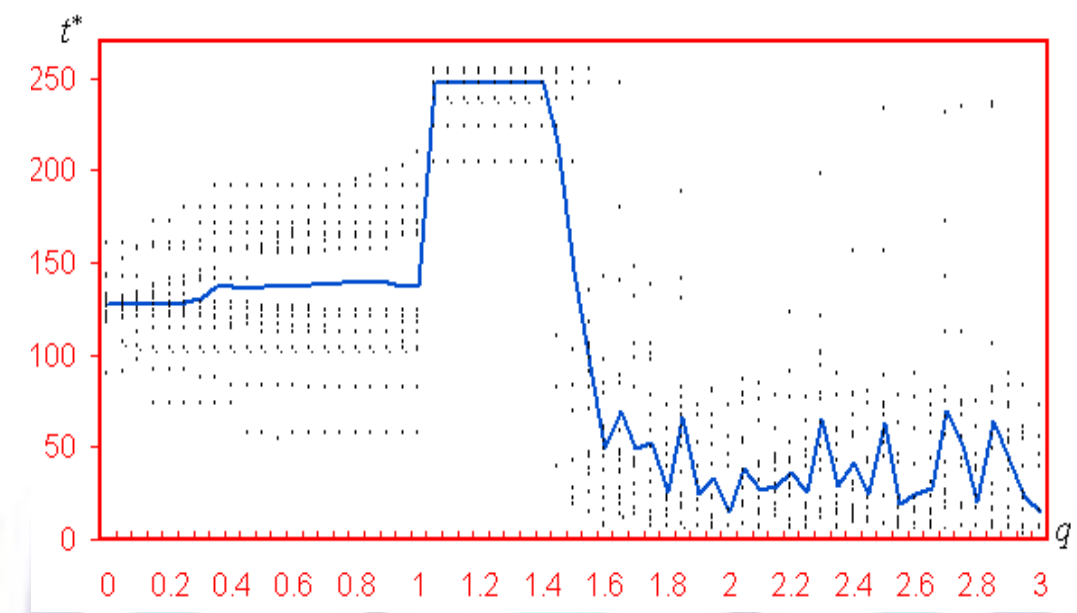

Figure 2. chart of average values of $t^{*}$ at various $\mathrm{q}$ of the test images.

original image $\quad t^{*}=132, q=0.1 \quad t^{*}=58, q=1$
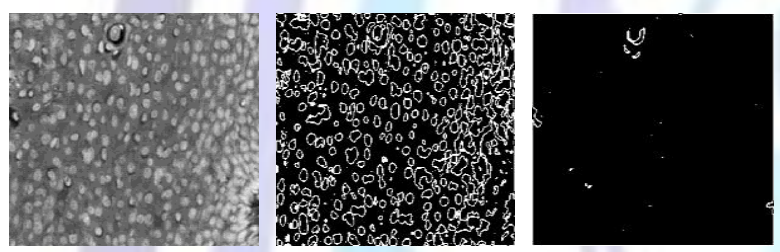

Figure 3. analskin image, and its the edge images.

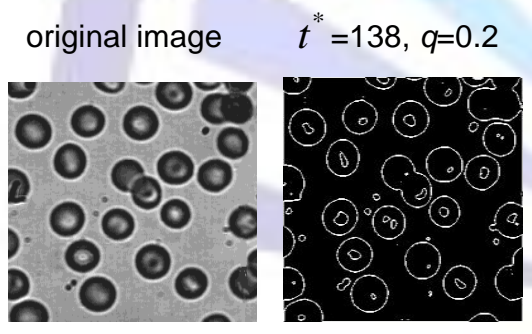

Figure 5. blood1 image, and its the edge images.

original image

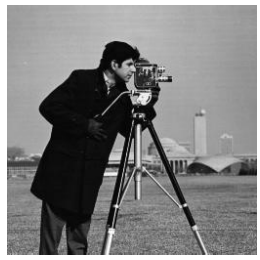

$t^{*}=123, q=0.2$

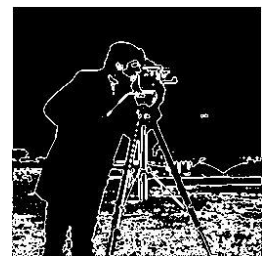

$t^{*}=192, q=1$

Figure 7. cameraman image, and its the edge images.

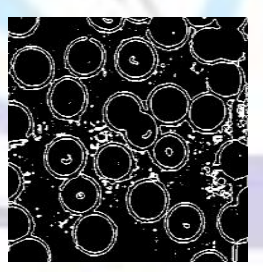

original image

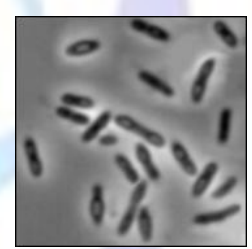

$t^{*}=104, q=0.4$

$t^{*}=104, q=1$

Figure 4. bacteria image, and its the edge images.

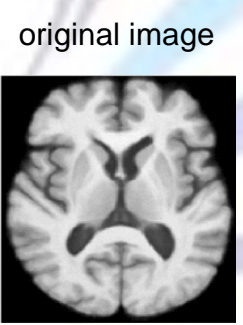

$t^{*}=155, q=0.5$

$t^{*}=172, q=1$
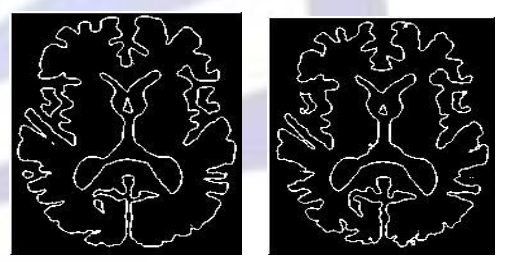

Figure 6. brain image, and its the edge images.

original image

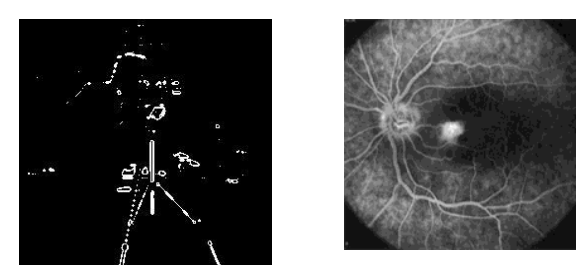

$t^{*}=117, q=0.4$

$t^{*}=113, q=1$
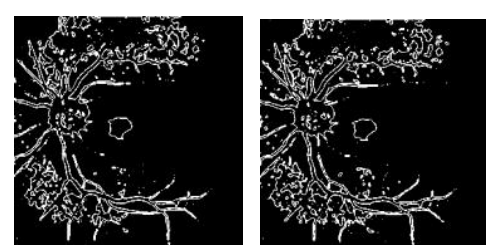

Figure 8. cell image, and its the edge images. 
original image $\quad t^{*}=158, \mathrm{q}=0.5 \quad t^{*}=210, \mathrm{q}=1$
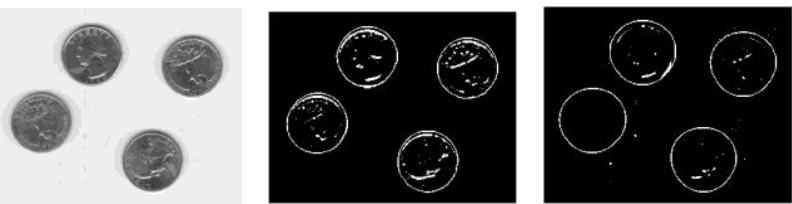

Figure 9. coins image, and its the edge images.

original image

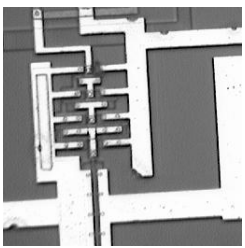

$t^{*}=125, q=0.1$

$t^{*}=83, q=1$
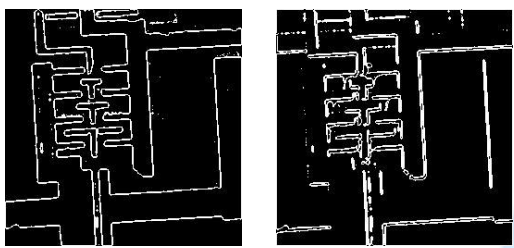

Figure 11. ic image, and its the edge images.

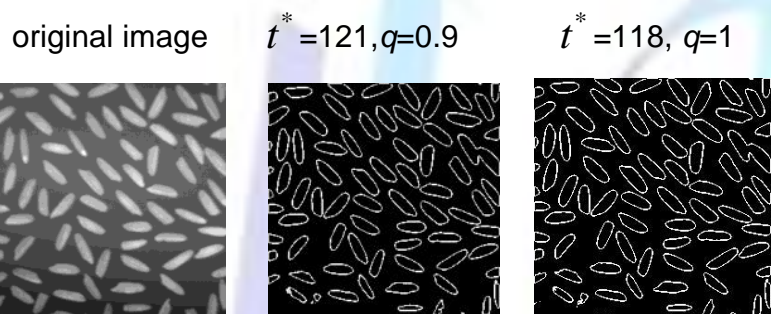

Figure 13. rice image, and its the edge images.

original image $t^{*}=131, q=0.05$
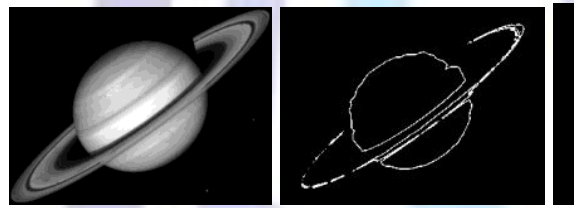

$t^{*}=165, q=1$

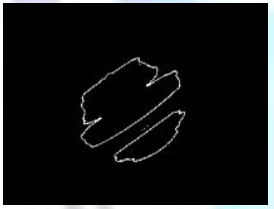

Figure 15. Saturn image, and its the edge images.

original image $\quad t^{*}=120, q=0.05 \quad t^{*}=180, q=1$
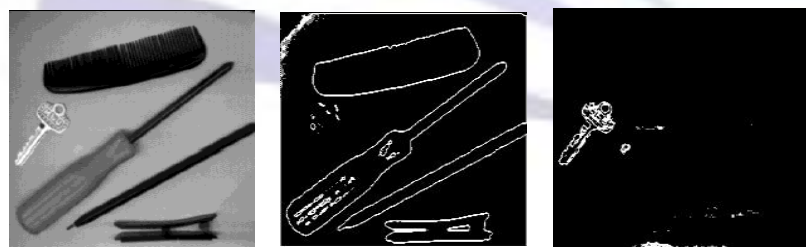

Figure 17. things image, and its the edge images. original image

$t^{*}=97, \mathrm{q}=0.1$

$t^{*}=121, \mathrm{q}=1$
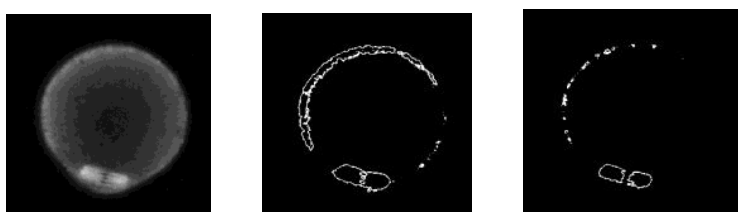

Figure 10. eggspindle image, and its the edge images.

original image

$t^{*}=99, q=1.7$

$t^{*}=167, q=1$
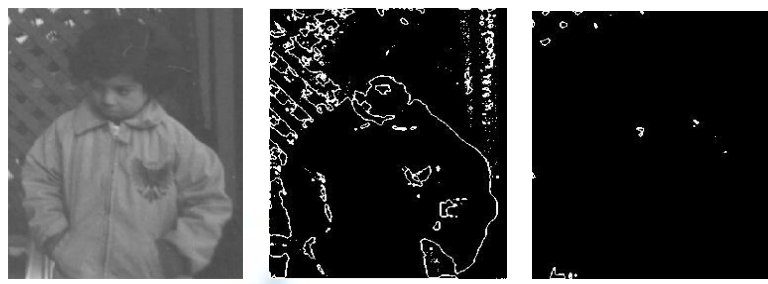

Figure 12. pout image, and its the edge images.

original image $\quad t^{*}=132, q=0.1 \quad t^{*}=103, q=1$
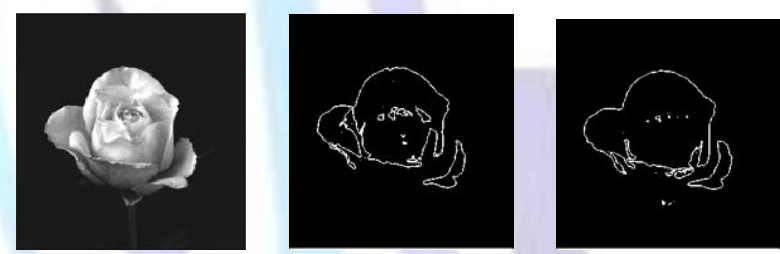

Figure 14. rose image, and its the edge images.

original image

$t^{*}=180, q=1.65$

$t^{*}=113, q=1$
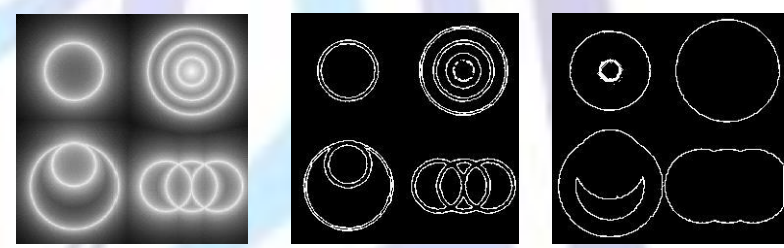

Figure 16. synthetic image, and its the edge images.

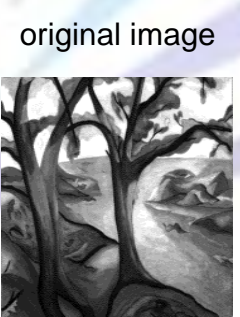

$t^{*}=125, q=0.4$

$t^{*}=124, q=1$

Figure 18. trees image, and its the edge images.

\section{CONCLUSION}

In this study, an attempt is made to develop a new technique for edge detection based on Tsallis entropy. It is already pointed out in the introduction that the traditional methods gives rise to the exponential increment of computational time. However, the proposed method is decrease the computation time with generate suitable quality of edge detection . Experiment results have demonstrated that the proposed scheme for edge detection works satisfactorily for different gray level digital images. The theoretical principles and systematic development of the algorithm for the proposed versatile edge detector is described in detail. The Tsallis $q$ coefficient can be used as an adjustable value and can play an important role as a tuning parameter in the image processing chain for the same class of images. This can be an advantage when the image processing tasks depend on an automatic thresholding. The technique has potential future in the field of digital image processing. 


\section{REFERENCES}

[1] M. P. de Albuquerque, I. A. Esquef, A.R. Gesualdi Mello, "Image Thresholding Using Tsallis Entropy." Pattern Recognition Letters 25 (2004) 1059-1065

[2] T. Chen, "An Edge Detection Algorithm Using Smoothing Functions", ICGST, GVIP Special Issue on Edge Detection , (2007)13-20,

[3] Hassanien, "Fuzzy Rough Sets Hybrid Scheme for Breast Cancer Detection", Intl. J. Image Vision Comput., 25 (2007)172-183.

[4] J.N. Kapur, P.K. Sahoo, Wong, A.K.C. "A new method for gray-level picture thresholding using the entropy of the histogram." Comput. Vision Graphics Image Process. 29, (1985) 273-285.

[5] F. Luthon, M. Lievin and F. Faux, "On the use of entropy power for threshold selection." Int. J. Signal Proc., 84 (2004)1789-1804.

[6] B. Mitra, , "Gaussian Based Edge Detection Methods- A Survey ". IEEE Trans. on Systems, Manand Cybernetics , 32 (2002) 252-260,.

[7] M. Roushdy, "Comparative Study of Edge Detection Algorithms Applying on the Grayscale Noisy Image Using Morphological Filter", GVIP, Special Issue on Edge Detection, (2007) 51-59.

[8] C.E. Shannon, "A Mathematical Theory of Communication", Int. J. Bell. Syst. Technical, 27, (1948) 379-423.

[9] P.K. Sahoo, S. Soltani, A.K.C. Wong, Y.C. Chen, "A Survey of the Thresholding Techniques", Computer Vision Graphics Image Process. 41 (1988) 233-260.

[10] B. Singh and A. P. Singh, " Edge Detection in Gray Level Images Based on the Shannon Entropy", J. Computer Sci., 4 (3) (2008)186-191.

[11] Y. Tsujimura and M. Gen, "Entropy-based Genetic Algorithm for Solving TSP" , In: IEEE Proceedings of the KES'98 April 21-23,2 (1998) 285-290.

[12] C. Tsallis, "Possible generalization of Boltzmann-Gibbs statistics," J. Stat. Phys., 52, (1988) 479-487.

[13] M. Wang and Y. Shuyuan, "A Hybrid Genetic Algorithm Based Edge Detection Method for SAR Image", In: IEEE Proceedings of the Radar Conference'05 May 9-12, 1(2005)503-506.

[14] Y. Yu and C. Chang. "A New Edge Detection Approach Based on Image Context Analysis". Int. J. Image Vision Comput, 24, (2006)1090-1102.

[15] R. Zhang, G. Zhao and L. Su, "A New Edge Detection Method in Image Processing", In: IEEE Proceedings of the ISCIT'05 Oct. 12-14, 1(2005) 445-448.

Mohamed A. El-Sayed has obtained his PhD degree in Computer Science in 2007 from Minia University, Egypt. His research interests include image processing, and graph drawing. He is the author of several articles published in reputed journals and is a member of different working groups. He is a lecturer in Math. Department, Faculty of Science, Fayoum University, Egypt. He is presently employed as Computer Science Assistant Professor at Computers and Information Technology College, Taif University, KSA. 